- 研究报告・

\title{
青岛嵘山湾人工鱼礁区底层游泳动物 群落结构特征
}

\author{
刘鸿赝 杨超杰 张沛东 李文涛 杨晓龙 张秀梅*
}

(中国海洋大学海水养殖教育部重点实验室, 山东青岛 266003)

\begin{abstract}
摘要: 基于 2015 年 5 月至 2016 年 1 月在青岛崂山湾人工鱼礁区的调查数据, 采用Margalef种类丰富度指数 $(d)$ 、 Shannon-Wiener多样性指数 $\left(H^{\prime}\right)$ 、Pielou均匀度指数 $(J)$ 、丰度/生物量曲线、等级聚类分析、SIMPER相似性百分比 分析和非度量多维尺度排序(NMDS)分析, 对底层游泳动物群落结构和多样性进行研究。调查期间共捕获游泳动物 61 种，隶属34科，其中鱼类36种，甲壳类22种，头足类3种。优势种为日本䌧 (Charybdis japonica)、许氏平鲉 (Sebastes schlegelii)、大泷六线鱼(Hexagrammus otakii)、斑头鱼(H. agrammus)和星康吉鳗(Conger myriaster), 其中 日本蟳 $(26.91 \%)$ 和许氏平鳋 $(26.74 \%)$ 的生物量占绝对优势。礁区游泳动物的渔获量和渔获种类数均多于对照区, 月 平均单位捕捞努力量渔获量 (CPUE) 最高值出现在 5 月, 达到735.74 $\pm 316.59 \mathrm{~g} \cdot \mathrm{net}^{-1} \cdot \mathrm{d}^{-1}$ 。游泳动物群落组成的特征 值上, 7、8月的多样性指数较高, 但鱼礁区和对照区无显著差异 $(P>0.05)$ 。丰度/生物量曲线表明, $5 、 6$ 月游泳动物 群落处于严重受干扰状态, 11 月和次年1月群落处于相对稳定状态。聚类分析表明, 除1月外, 各月份不同区域的样 方相似性都较高, 5、6月三亩前礁区的相似性最高, 达到 $86.21 \%$ 。
\end{abstract}

关键词: 人工鱼礁; 游泳动物群落结构; 多样性; 丰度/生物量曲线; 崂山湾

\section{Demersal nekton community structure of artificial reef zones in Laoshan Bay, Qingdao}

Hongyan Liu, Chaojie Yang, Peidong Zhang, Wentao Li, Xiaolong Yang, Xiumei Zhang* Key Laboratory of Mariculture, Ministry of Education, Ocean University of China, Qingdao, Shandong 266003

\begin{abstract}
A field survey of the artificial reef and the control zones in Laoshan Bay, Shandong Province was performed from May 2015 to January 2016. We analyzed the community structure and biodiversity of demersal nekton by using Margalef's species richness index, Shannon-Wiener diversity index, Pielou's evenness index, abundance-biomass comparison curves, clustering analysis, SIMPER (similarity percentage) analysis, and Non-metric Multi Dimensional Scaling (NMDS). A total number of 61 species of nekton belonging to 34 families were captured during the surveys, including 36 species of fish, 22 species of crustaceans and 3 species of cephalopods. Charybdis japonica, Sebastes schlegelii, Hexagrammus otakii, $H$. agrammus and Conger myriaster were the dominant species, of which C. japonica and S. schlegelii were the dominant species by weight $(26.91 \%$ and $26.74 \%$, respectively). The highest monthly mean catch per unit effort (CPUE) in the investigated zones appeared in May 2015, and reached up to $735.74 \mathrm{~g} \cdot$ net $^{-1} \cdot \mathrm{d}^{-1}$. It was noted that the diversity indices were higher in July and August, but there were no significant differences in community structure parameters between the artificial reef and the control zones $(P>0.05)$. The abundance-biomass curves $(\mathrm{ABC})$ of the nekton community indicated that the community was seriously disturbed in May and June 2015, but was stable by November 2015 and January 2016. Cluster analysis showed that similarities were high in different months and different zones, except January 2016, and the highest similarity appeared in the artificial reef of the Sanmuqian zone between May and June 2015, when it reached $86.21 \%$.
\end{abstract}

收稿日期: 2016-04-25; 接受日期: 2016-07-19

基金项目: 国家海洋公益性行业科研专项(201405010; 201305043)和国家自然科学基金(31572227)

* 通讯作者 Author for correspondence. E-mail: gaozhang@ouc.edu.cn 
Key words: artificial reef; nekton community structure; diversity; abundance-biomass comparison curves; Laoshan Bay

青岛崂山湾鱼类产卵场修复区位于黄山头至 崂山头之间的近岸水域，该区域湾岸陡峭，曾经海 藻繁茂, 是鱼、虾洄游的必经之路, 也是近岸岩礁 性鱼类的重要产卵场。为开展海参、鲍鱼等资源增 殖与保护，2006-2014年间该海域进行了大规模人 工鱼礁投放工作。人工鱼礁是指利用鱼类等海洋生 物的趋性(如趋触性、趋流性等), 为了聚集鱼类和其 他海洋生物而在海域中设置的人工构造物 (Rounsefell，1972)。人工鱼礁能够在投放海域产生 上升流、浴流等多种流态, 底质中的一些营养物质 进入水体，从而使水体中的营养盐得到补充、优化, 促进饵料生物的生长、繁殖, 最终通过生物链的传 递来影响渔业资源(陈勇等, 2002; 林军和章守宇, 2006; 周艳波等, 2011)。崂山湾海域水体环境优良, 栖息地结构复杂, 人工鱼礁的投放进一步提高了海 区的空间异质性，随着礁体附着生物群落的逐步形 成, 使得该海域成为游泳动物优良的栖息和繁殖场 所。近年来, 有关崂山湾及其邻近海域的研究主要 涉及底栖大型海藻(唐衍力等, 2012)、浮游植物(王 欣等, 2014)、鱼类群落的摄食生态(张波等, 2015) 以 及一些放流种的增殖效果评价(梅春等, 2010; 袁伟 等, 2015)等, 而有关人工鱼礁区底层游泳动物群落 结构特征及多样性的研究尚未见报道。游泳动物在 海洋生态系统中处于较高层次, 海域游泳动物群落 结构的研究在海洋生态学、渔业生产以及渔业管理 等方面具有重要意义(李凡等, 2013)。

本研究以青岛崂山湾岩礁性鱼类产卵场综合 修复示范区的人工鱼礁区和对照区为研究区域，通 过对底层游泳动物群落和主要环境因子的监测, 分 析鱼礁区和对照区底层游泳动物群落的结构特征 和多样性，旨在探索人工鱼礁的投放对海域生物资 源组成的影响, 为系统全面地评价人工鱼礁的生态 功能及其对渔业资源的修复效果提供参考依据。

\section{1 材料和方法}

\section{1 取样地点}

青岛崂山湾产卵场修复示范区 $\left(36^{\circ} 08.49^{\prime}-\right.$ $36^{\circ} 10.02^{\prime} \mathrm{N}, 120^{\circ} 41.32^{\prime}-120^{\circ} 42.99^{\prime} \mathrm{E}$ )由黄山口、三
亩前和列坡 3 个海区组成, 2006年开始建礁, 2014年 春建设完毕，共计投石 3.2 万空方，形成礁区 5 处， 面积 39.7 ha。

本研究设置的取样点如图1所示, 从北向南划 分为黄山口礁区 $(H 区)$ 、对照区 $(C 区)$ 和三亩前礁区 (S区) 3 个区域，每个区域设置4个取样点。

\section{2 样品采集与分析}

2015年5-11月和2016年1月使用地笼网采集游 泳动物。地笼网规格: 中间部位为 21 个 $25 \mathrm{~cm} \times 40$ $\mathrm{cm}$ 的钢筋框, 两端为 2 个 $20 \mathrm{~cm} \times 30 \mathrm{~cm}$ 的钢筋框, 钢筋框间距为 $25 \mathrm{~cm}$, 进鱼口纵深 $20 \mathrm{~cm}$, 网目规格 $18 \mathrm{~mm}$ 。每 5 个地笼网连接构成一个网笼单元(全长 $30 \mathrm{~m})$, 每个取样点设置 1 个网笼单元。地笼网与岸 基平行放置, 放置时间为 3 天左右。在实验室内对所 采集的渔获进行鉴定分类, 并对样品进行称量和计 数。采集游泳动物的同时, 利用多参数水质检测仪 监测鱼礁区和对照区的水温(temperature)、水深 (deep)、盐度(salinity)、溶解氧(dissolved oxygen)和 $\mathrm{pH}$ 等环境因子。样品采集、保存及分析均按《海洋 调查规范》(GB/T12763-2007)和《海洋监测规范》 (GB17378-1998)进行。

游泳动物的学名及分类地位以 《中国鱼类系统 检索》(成庆泰和郑葆珊, 1987)为依据, 并参考FAO FishBase数据库(Palomares \& Pauly, 2015); 鱼类生 物的适温类型参考刘静和宁平(2011)的研究, 包括 暖温种 (warm temperature species, WT)、暖水种 (warm water species, WW)、冷温种 (cold temperature species, CT) 以及冷水种 (cold water species, CW); 甲 壳类和头足类的学名及分类地位以 《中国海洋生物 名录》(刘瑞玉, 2008)和《中国北部无脊椎动物》(杨 德渐和王永良, 1989)为依据, 并参照SeaLifeBase数 据库(Palomares \& Pauly, 2015)。

\section{3 数据处理与分析}

将每个网笼单元的渔获物作为一个样本来进 行数据分析, 分析前对原始数据作标准化处理, 用 每天每网笼单元的渔获量 $\left(\mathrm{g} \cdot \mathrm{net}^{-1} \cdot \mathrm{d}^{-1}\right)$ 来表示地笼网 的单位捕捞努力量(catch per unit effort, CPUE) (吴 忠金等, 2012)。采用Shannon-Wiener多样性指数 $\left(H^{\prime}\right)$ 


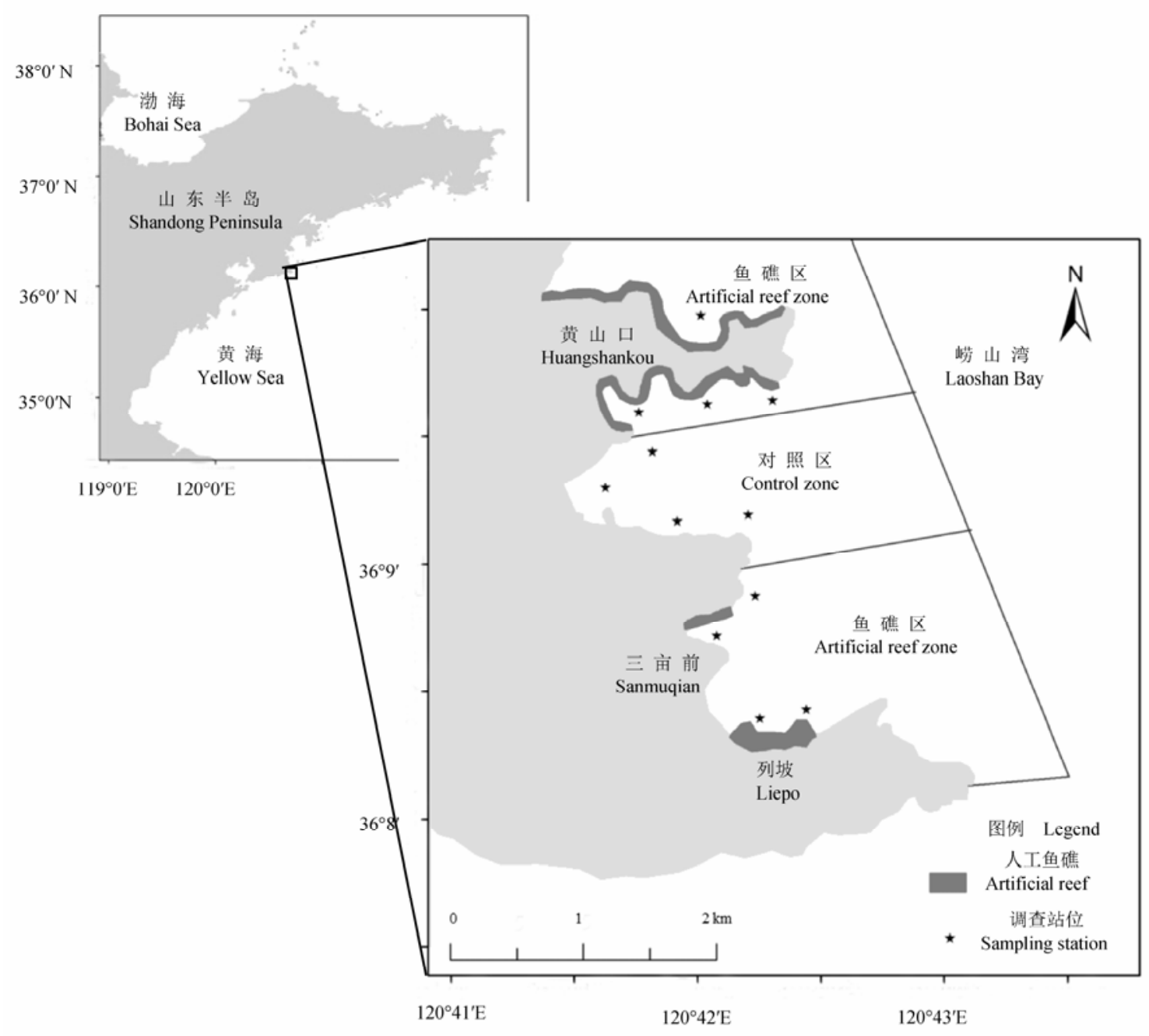

图1 崂山湾鱼礁区和对照区游泳动物调查站位

Fig. 1 Sampling stations for the nekton investigation in the artificial reef zones and the control zones in Laoshan Bay

(Ludwig \& Reynolds, 1988)、Margalef种类丰富度指 数 $(d)$ (Margalef, 1968)、Pielou均匀度指数 $(J)$ (Pielou, 1966)以及Pinkas相对重要性指数(IRI) (Pinkas et al, 1971)对渔获数据进行分析。各指数的计算公式如 下:

Shannon-Wiener多样性指数:

$$
H^{\prime}=-\sum_{\mathrm{i}=1}^{\mathrm{s}} P_{\mathrm{i}} \log _{2} P_{i}
$$

Margalef种类丰富度指数:

$$
d=(S-1) / \log _{2} N
$$

Pielou均匀度指数：

$$
J=H^{\prime} / \log _{2} S
$$

Pinkas相对重要性指数(IRI):

$$
\mathrm{IRI}=(N \%+W \%) \times F \%
$$

式中, $S$ 为游泳动物种类数; $N$ 为游泳动物总个体数; $P_{i}$ 表示第 $i$ 种游泳动物的渔获量占总渔获量的百分 比; $N \%$ 为某一游泳动物的渔获尾数占总渔获尾数 的百分比; $W \%$ 为某一游泳动物的生物量占总生物 量的百分比; $F \%$ 为某一游泳动物出现的站位数占 总站位数的百分比。定义IRI $>1,000$ 的种类为优势种, $100 \leq \mathrm{IRI}<1,000$ 的种类为重要种, 优势种和重要种 统称主要种(李凡等, 2013)。

利用ABC (abundance-biomass comparison)曲线 和W统计(W-statistic) (Warwick, 1986)描述群落稳定 性以及生物量/丰度的优势地位。以各月份各调查站 位渔获量为原始矩阵，进行平方根转换以后，利用 等级聚类 (CLUSTER) 和非度量多维尺度排序 
(NMDS) 分析各月份各调查区域游泳动物的组成特 征。应用SIMPER相似性百分比分析游泳动物对站 位组内相似性和组间差异性的贡献率, 将贡献率 $3 \%$ 以上的游泳动物定为主要特征种类(徐炳庆等, 2011)。采用Stress系数检验NMDS结果的可信度: Stress $<0.1$ 时结果具有较好的解释意义; $0.1 \leq$ Stress $<0.2$ 时结果具有一定的解释意义; Stress $\geq 0.2$ 时结果不具解释意义 (马藏允和王惠卿, 1997; Clarke \& Warwick, 2001)。利用Bray-Curtis相 异性指标(Bray \& Curtis, 1957)分析各调查月份之间 渔获种类资源量组成的差异, 并作出不同站位相似 度树状图。

使用Excel 2010、SPSS 19.0和PRIMER 6.0软件 对相关数据进行分析, 利用单因素方差分析不同区 域间的差异，显著性水平为 $\alpha=0.05$ 。图件绘制使用 OriginPro 8和PRIMER 6.0软件, 使用Arcgis 10.2 软 件完成站位布设图的绘制。

\section{2 结果}

\section{1 水域环境特征}

对调查区不同月份底层水体环境因子进行方 差分析, 结果表明, 不同月份的底层水体环境因子 变化显著(表1)。水温和溶解氧含量之间具有相反的 变化趋势, 5 月水温较低, 但溶解氧含量最高, 8 月水 温最高, 而溶解氧含量最低; 不同月份之间的盐度 变化较小, 但有一定的季节变化趋势, 盐度在 5 月 最高, 11 月最低; $\mathrm{pH}$ 最大值出现在11月, 最小值出现 在7月。

\section{2 游泳动物种类组成和单位捕捞努力量(CPUE)}

2015年5月至2015年11月以及 2016 年1月 8 个月 份的调查共捕获游泳动物 34 科 61 种(附录 1 ), 其中鱼
类 18 科 36 种，甲壳类 14 科 22 种，头足类仅有长蛸 (Octopus variabilis)、短蛸 $(O$. ocellatus $)$ 和金乌贼 (Sepia esculenta) 等2科3种。捕获的36种鱼类生物的 适温类型均为暖温种和冷温种, 无冷水种和暖水 种。暖温种主要包括星康吉鳗(Conger myriaster)、 花鲈(Lateolabrax maculatus)、褐牙鲆(Paralichthys olivaceus)等 27 种, 占鱼类总种数的 $75 \%$, 冷温种包 括许氏平鲉(Sebastes schlegelii)、大泷六线鱼 (Hexagrammus otakii)、斑头鱼(H. agrammus)等9种, 占鱼类总种数的 $25 \%$ 。各调查航次地笼网的 CPUE 显示(图2)，地笼网的月平均 CPUE为515.35 $309.47 \mathrm{~g} \cdot \mathrm{net}^{-1} \cdot \mathrm{d}^{-1}, 5$ 月份最高, 为735.74 \pm 316.59 $\mathrm{g} \cdot \mathrm{net}^{-1} \cdot \mathrm{d}^{-1}, 1$ 月份最低，仅为 $161.59 \pm 185.02$ $\mathrm{g} \cdot \mathrm{net}^{-1} \cdot \mathrm{d}^{-1}$, 各航次CPUE具有显著性差异 $(P<0.05)$ 。 黄山口礁区共捕获游泳动物 50 种, 月平均CPUE为 $563.705 \pm 356.12 \mathrm{~g} \cdot \mathrm{net}^{-1} \cdot \mathrm{d}^{-1}$, 三亩前礁区共捕获游 泳动物 41 种, 月平均 CPUE为566.911 \pm 262.99 $\mathrm{g} \cdot \mathrm{net}^{-1} \cdot \mathrm{d}^{-1}$, 对照区仅捕获游泳动物 38 种, 月平均

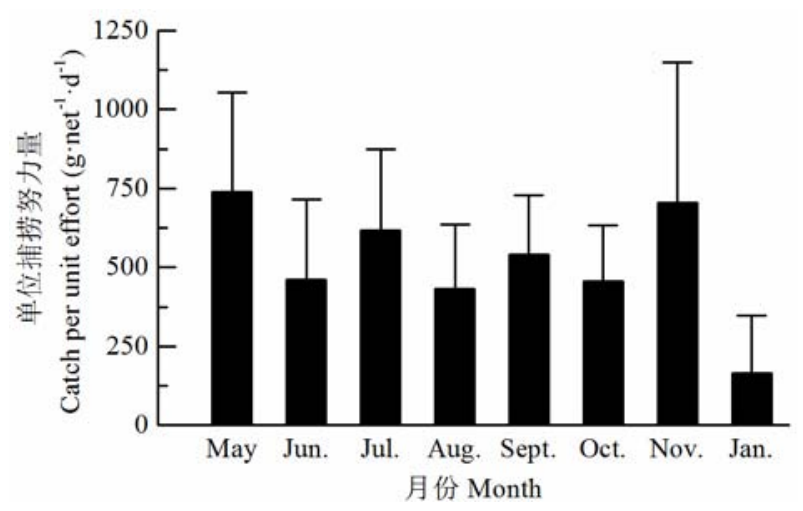

图2 单位捕捞努力量均值的月变化

Fig. 2 Variation in mean catch per unit fishing effort

表1 崂山湾人工鱼礁区底层水域环境特征

Table 1 Environmental conditions of bottom water of the artificial reef zones in Laoshan Bay

\begin{tabular}{|c|c|c|c|c|c|c|c|c|}
\hline $\begin{array}{l}\text { 环境变量 } \\
\text { Environment } \\
\text { variable }\end{array}$ & $\begin{array}{l}\text { 1月 } \\
\text { Jan. }\end{array}$ & $\begin{array}{l}\text { 5月 } \\
\text { May }\end{array}$ & $\begin{array}{l}\text { 6月 } \\
\text { Jun. }\end{array}$ & $\begin{array}{l}\text { 7月 } \\
\text { Jul. }\end{array}$ & $\begin{array}{l}\text { 8月 } \\
\text { Aug. }\end{array}$ & $\begin{array}{l}\text { 9月 } \\
\text { Sept. }\end{array}$ & $\begin{array}{l}\text { 10月 } \\
\text { Oct. }\end{array}$ & $\begin{array}{l}\text { 11月 } \\
\text { Nov. }\end{array}$ \\
\hline $\begin{array}{l}\text { 水温 } \\
\text { Temperature }\left({ }^{\circ} \mathrm{C}\right)\end{array}$ & $4.57 \pm 0.12^{\mathrm{a}}$ & $11.93 \pm 0.23^{\mathrm{b}}$ & $14.09 \pm 0.08^{c}$ & $22.68 \pm 0.51^{\mathrm{d}}$ & $25.84 \pm 0.11^{\mathrm{e}}$ & $24.70 \pm 0.06^{\mathrm{f}}$ & $20.30 \pm 0.15^{\mathrm{g}}$ & $14.09 \pm 0.05^{\mathrm{c}}$ \\
\hline $\begin{array}{l}\text { 溶解氧含量 } \\
\text { DO (mg/L) }\end{array}$ & $8.33 \pm 1.45^{\mathrm{a}}$ & $9.18 \pm 0.78^{\mathrm{ab}}$ & $7.89 \pm 0.84^{\mathrm{b}}$ & $6.04 \pm 0.33^{\mathrm{c}}$ & $4.22 \pm 0.43^{\mathrm{d}}$ & $4.67 \pm 0.03^{\mathrm{de}}$ & $6.71 \pm 0.05^{\mathrm{c}}$ & $5.78 \pm 0.02 \mathrm{~d}^{\mathrm{ce}}$ \\
\hline 盐度 Salinity & $31.29 \pm 0.20^{\mathrm{a}}$ & $31.87 \pm 0.06^{\mathrm{b}}$ & $31.19 \pm 0.07^{b}$ & $30.96 \pm 0.03^{\mathrm{c}}$ & $30.86 \pm 0.04^{\mathrm{c}}$ & $30.99 \pm 0.03^{\mathrm{c}}$ & $31.16 \pm 0.03^{\mathrm{b}}$ & $30.51 \pm 0.15^{\mathrm{d}}$ \\
\hline $\mathrm{pH}$ & $7.80 \pm 0.12^{\mathrm{a}}$ & $7.70 \pm 0.03^{b}$ & $7.85 \pm 0.05^{\mathrm{c}}$ & $7.59 \pm 0.03^{\mathrm{a}}$ & $7.77 \pm 0.08^{\mathrm{b}}$ & $7.73 \pm 0.04^{b}$ & $7.70 \pm 0.03^{\mathrm{ab}}$ & $7.89 \pm 0.07^{\mathrm{d}}$ \\
\hline
\end{tabular}

同行间相同的小写字母表示差异不显著 $(P>0.05)$

Means with same lower case superscript letter in the same row were no significant difference $(P>0.05)$ 
表2 2015年5月至2016年1月崂山湾主要游泳动物的Pinkas相对重要性指数(“一”表示未捕获)

Table 2 Index of relative importance (IRI) of the main nektons in Laoshan Bay from May 2015 to January 2016 (“- " means not catch)

\begin{tabular}{|c|c|c|c|c|c|c|c|c|}
\hline 种类 & 5月 & 6月 & 7月 & 8月 & 9月 & 10月 & 11月 & 1月 \\
\hline Species & May & Jun. & Jul. & Aug. & Sept. & Oct. & Nov. & Jan. \\
\hline 许氏平鲉 Sebastes schlegelii & 4,665 & 2,671 & 1,829 & 847 & 2,413 & 2,935 & 10,485 & 7,963 \\
\hline 日本蟳 Charybdis japonica & 2,045 & 2,161 & 7,852 & 8,158 & 7,728 & 9,420 & 2,386 & 189 \\
\hline 大泷六线鱼 Hexagrammus otakii & 5,288 & 6,303 & 1,794 & 734 & 1,522 & 1,467 & 1,406 & 2,065 \\
\hline 斑头鱼 Hexagrammus agrammus & 1,601 & 3,168 & 75 & 530 & 350 & 518 & 1,065 & 339 \\
\hline 星康吉鳗 Conger myriaster & 394 & 134 & 1,372 & 807 & 1,290 & 1,103 & 747 & 13 \\
\hline 寄居蟹科 Paguridae & 1,045 & 1,206 & 2,496 & 1,957 & 24 & 46 & - & 642 \\
\hline 褐菖鲉 Sebastiscus mamoratus & 27 & 93 & 31 & 138 & 871 & 2,087 & 1,567 & 199 \\
\hline 朝鲜平鲉 Sebastes koreanus & 691 & 616 & 30 & 10 & 330 & 359 & 225 & 47 \\
\hline 长蛸 Octopus variabilis & 616 & 69 & 45 & - & 159 & 683 & 95 & 191 \\
\hline 厚头平鲉 Sebastes pachycephalus & 281 & 328 & - & 17 & 69 & 195 & 154 & 79 \\
\hline 口虾蛄 Oratosquilla oratoria & 23 & 34 & 398 & 418 & 1,041 & 188 & 158 & - \\
\hline 敖氏长臂虾 Palaemon ortmanni & 53 & 32 & 19 & 150 & 47 & 225 & 61 & - \\
\hline 繸鳚 Chirolophis japonicus & 167 & 4 & - & - & - & - & - & 23 \\
\hline 方氏云鳚 Pholis fangi & 4 & 2 & - & - & 33 & 25 & 503 & - \\
\hline 花鲈 Lateolabrax maculatus & - & - & 10 & - & 588 & 117 & 787 & - \\
\hline 强壮菱蟹 Parthenope validus & - & - & 49 & 185 & 518 & - & - & - \\
\hline 三疮梭子蟹 Portunus trituberculatus & - & - & - & 5 & 1,107 & - & 31 & - \\
\hline 黄姑鱼 Nibea albiflora & - & - & - & 40 & 519 & 34 & - & - \\
\hline 银姑鱼 Pennahia argentata & - & - & - & - & 205 & - & - & - \\
\hline 褐牙鲆 Paralichthys olivaceus & - & - & 16 & 4 & 436 & - & - & - \\
\hline
\end{tabular}

CPUE为417.06 $\pm 272.99 \mathrm{~g} \cdot \mathrm{net}^{-1} \cdot \mathrm{d}^{-1}$ ，显著低于鱼礁 区 $(P<0.05)$ 。

\section{3 游泳动物优势种组成及群落稳定性变化}

表2给出了各航次调查中IRI $\geq 100$ 的种类分布, 日本蟳 (Charybdis japonica)、许氏平鲉和大泷六线 鱼是崂山湾海域全年性优势种, 斑头鱼、星康吉鳗、 褐菖鲉(Sebastiscus mamoratus)和寄居蟹(Paguridae) 则属于季节性优势种，且优势种的优势度值在各月 份的调查中变化较大。优势种类在5、6、7月最多, 在 1 月最少。根据渔获量组成, 日本䌧的生物量最多, 其渔获量占总渔获量的 $26.91 \%$, 其次为许氏平鲉, 占 $26.74 \%$, 大泷六线鱼占 $10.40 \%$, 星康吉鳗占 $6.81 \%$, 斑头六线鱼占 $6.33 \%$, 其他种类占 $22.81 \%$ 。

游泳动物 $\mathrm{ABC}$ 曲线(图3)显示, 5 月和 6 月的丰度 曲线始终位于生物量曲线的上方, $\mathrm{W}$ 为负值, 丰度 比生物量更具优势地位, 群落优势种以小个体生物 为主, 群落处于严重受干扰状态; 7-10月份, 生物 量曲线和丰度曲线出现交叉重叠, 且 $\mathrm{W}$ 为正值, 群 落处于中度扰动状态；11月和1月份的生物量曲线 始终位于丰度曲线的上方, $\mathrm{W}$ 为正值, 生物量比丰
度更具优势地位，群落优势种以大个体生物为主, 群落处于较稳定状态。

\section{4 多样性分析}

各调查区域游泳动物群落的物种多样性指数、 种类丰富度指数和均匀度指数在不同月份之间存 在一定变化, 但是差异不显著 $(P>0.05)$ 。5月至9月, Shannon-Wiener多样性指数和Margalef种类丰富度 指数呈现增加的趋势，最大值分别出现在 9 月的黄 山口礁区和 8 月的三亩前礁区，分别达到 2.818 和 3.934；9月之后逐渐降低，至次年1月降到最低，最 低值出现在三亩前礁区，分别为 1.219 和 1.173 。均匀 度指数无明显的变化趋势，除 1 月以外，其他各月 无明显差异，最大值出现在1月的黄山口礁区，为 0.9156 , 最小值出现在 1 月的三亩前礁区，为 0.6805 。

\section{5 群落结构分析}

聚类分析结果表明(图5)，除1月外，各调查月 份样方间的相似性均达到 $55 \%$ 以上。相似性最高的 两个样方为 5 月和 6 月的三亩前礁区，相似度达到 $86.21 \%$ 。NMDS排序图(图6)与群落聚类分析相似度 

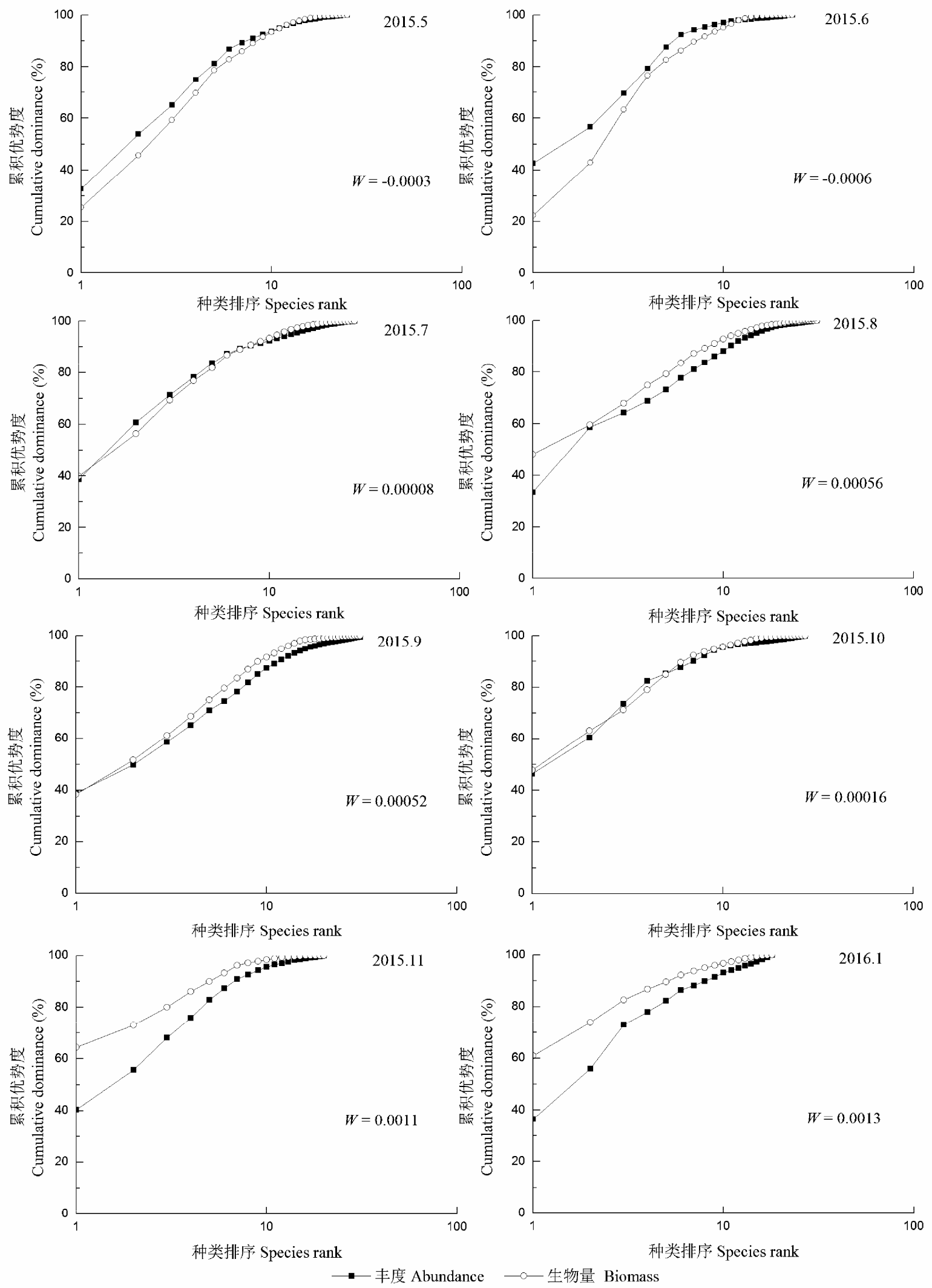

图32015年5月至2016年1月崂山湾游泳动物丰度/生物量曲线

Fig. 3 Abundance-biomass comparison curves of nektons in Laoshan Bay from May 2015 to January 2016 

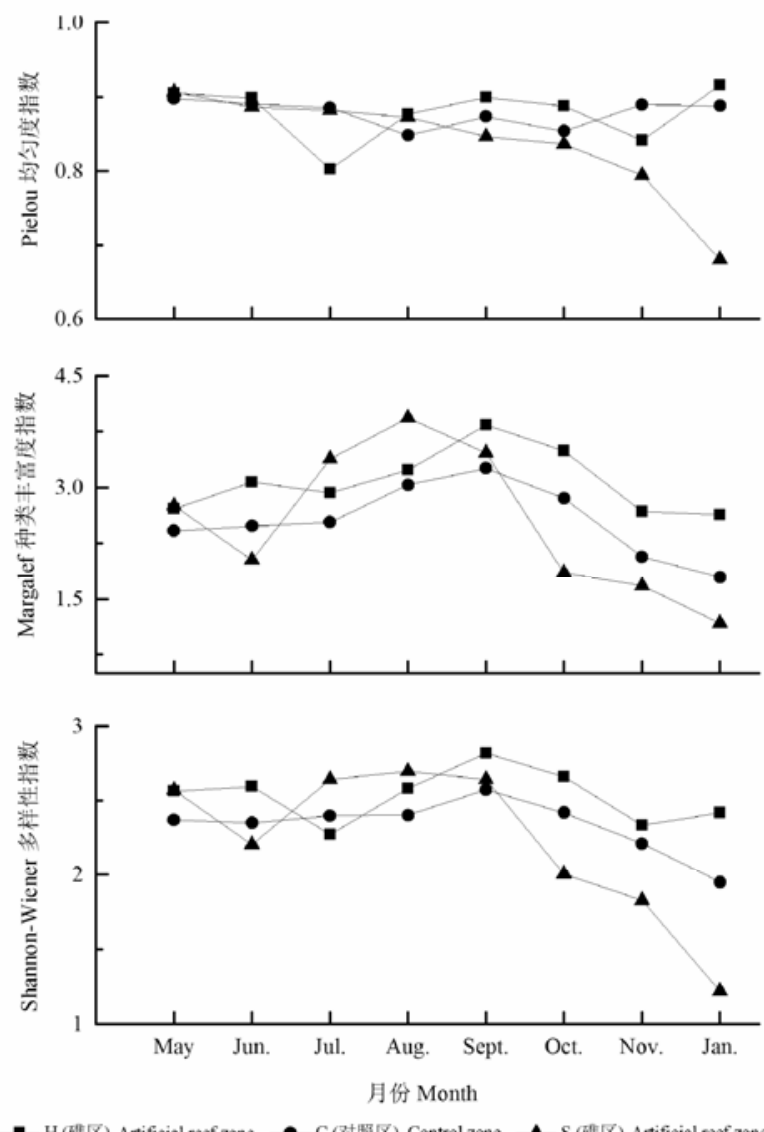

图4 崂山湾不同区域游泳动物群落多样性指数的季节变化 Fig. 4 Seasonal variations in the diversity of nekton community of different zones in Laoshan Bay

$55 \%$ 以上的结果一致, Stress值为 0.15 , 具有一定的 解释意义。

利用SIMPER相似性百分比分析各组内群落结 构相似的特征种以及组间群落差异的分歧种和贡 献率。黄山口礁区各调查网次渔获结构的平均相似 性为 $55.58 \%$, 特征种包括日本蟳、许氏平鲉、大泷 六线鱼、斑头鱼、星康吉鳗、厚头平鲉(Sebastes pachycephalus)、朝鲜平鲉(S. koreanus) 和褐菖鲉, 这 8 种渔获物对组内平均相似性的累计贡献率达 $88.39 \%$; 对照区各调查网次渔获结构的平均相似性 为 $63.80 \%$, 特征种包括许氏平鲉、日本蟳、大泷六 线鱼、星康吉鳗、长蛸、褐菖鲉和斑头鱼, 这7种渔 获物对组内平均相似性的累计贡献率达 $94.00 \%$; 三 亩前礁区各调查网次渔获结构的平均相似性为 $55.37 \%$, 特征种包括许氏平鲉、日本蟳、大泷六线
鱼、斑头鱼和褐菖鲉，这5种渔获物对组内平均相似 性的累计贡献率达 $91.94 \%$ 。黄山口礁区和对照区的 平均相异性为 $41.70 \%$ ，分歧种包括日本蟳、许氏平 鲉、斑头鱼、长蛸、星康吉鳗、厚头平鲉、寄居蟹 和大泷六线鱼，这 8 种渔获物对组间平均相异性的 累计贡献率达 $69.83 \%$; 黄山口礁区和三亩前礁区的 平均相异性为 $44.99 \%$, 分歧种包括许氏平鲉、日本 蟢、斑头鱼、星康吉鳗、长蛸、大泷六线鱼和寄居 蟹, 这 7 种渔获物对组间平均相异性的累计贡献率 达 $62.92 \%$; 对照区和三亩前礁区的平均相异性为 $39.83 \%$ ，分歧种包括日本䌧、许氏平鲉、长蛸、斑 头鱼、口虾蛄(Oratosquilla oratoria) 和星康吉鳗，这 6 种渔获物对组间平均相异性的累计贡献率达 $62.82 \%$ 。

\section{3 讨论}

\section{1 游泳动物种类组成}

青岛崂山湾产卵场修复示范区底层游泳动物 以鱼类为主, 周年地笼网调查出现的 36 种鱼类均是 我国黄渤海海域常见种。游泳动物优势种为日本 蟳、许氏平鲉、大泷六线鱼、斑头鱼以及星康吉鳗, 这与日照前三岛海域(焦金菊等, 2011)、山东朱旺港 海域人工鱼礁区(徐浩等, 2012)、荣成俚岛人工鱼礁 区(吴忠釒等, 2012)、马鞍列岛岩礁区域(汪振华等, 2012)以及威海西港(于晴和唐衍力，2015)等岩礁游 泳动物优势种相似。日本蟳作为一种典型的岩礁生 物，其索饵、繁殖、避敌等行为均在岩礁或洞穴中 进行(徐浩等, 2012); 许氏平鲉、大泷六线鱼、斑头 鱼均属于岩礁定居性鱼类，常栖息于海藻从及浅海 岩礁等光线较弱的地方(张硕等, 2008 ; 纪东平等, 2014，2015); 星康吉鳗属于岩礁非定居性鱼类，常 常栖息于泥沙海底及相邻的水下暗礁、岩洞等(张迎 秋，2015)。人工鱼礁投放以后，一些大型海藻附着 生长, 为鱼类提供了良好的栖息场所。此外, 鱼礁 所产生的阴影、水流等也会吸引鱼类聚集。

\section{2 游泳动物渔获量}

周年 8 个航次的调查结果显示，人工鱼礁区的 渔获量和渔获种类数均明显多于对照区。同时，春 季、秋季航次的鱼类生物量明显多于夏季和冬季。 分析认为, 礁区大型海藻资源丰富, 栖息地的复杂 程度高于对照区，吸引鱼类等生物在此聚集，从而 


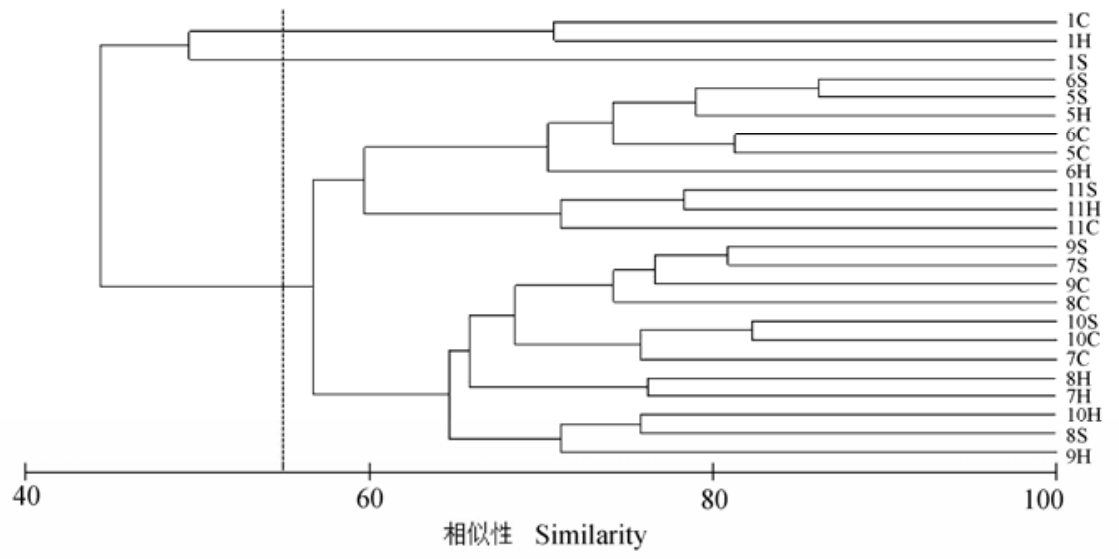

图5 崂山湾不同区域不同月份游泳动物群落聚类分析(数字表示月份, 字母表示区域)

Fig. 5 The cluster analysis of the nekton community in different months and different zones in Laoshan Bay. Digits indicate the month, and letters indicate zones.

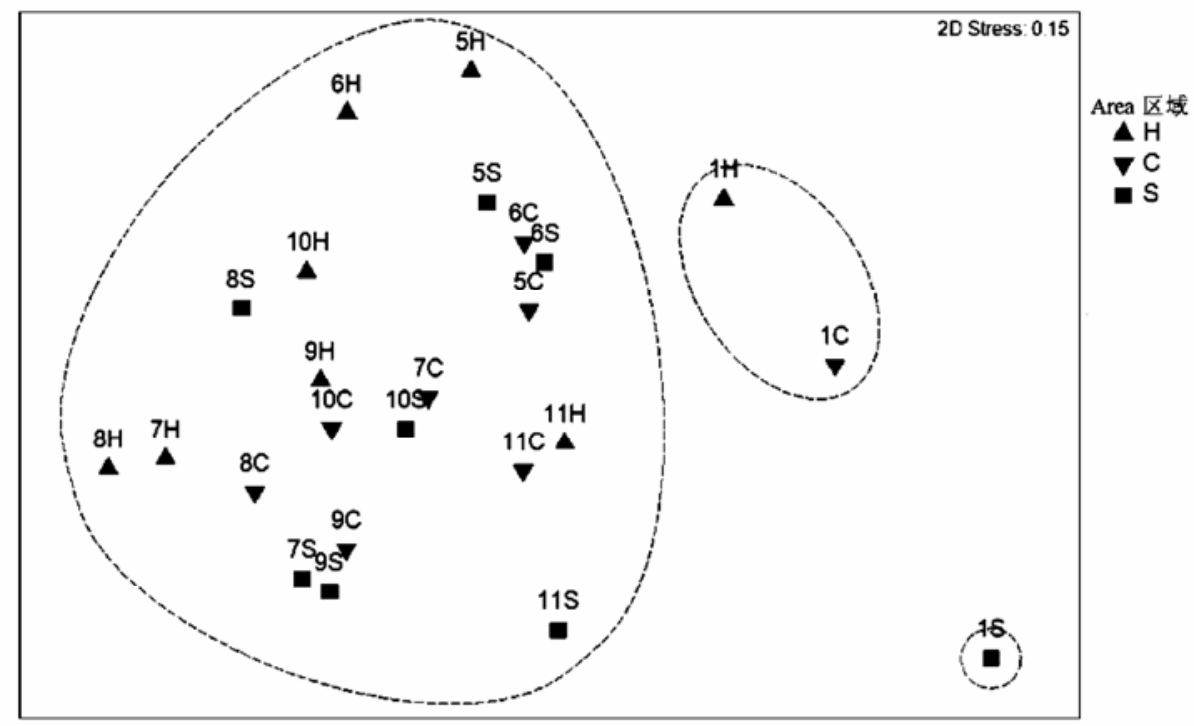

图6 苏山湾不同区域不同月份游泳动物群落NMDS排序分析。数字表示月份，字母表示区域。

Fig. 6 NMDS analysis of the nekton community in different months and different zones in Laoshan Bay. Digits indicate the month, and letters indicate zones.

导致礁区生物量明显多于对照区。青岛崂山湾是山 东近岸浅海重要的产卵场和幼鱼的索饵育肥场, 春 季水温回升, 一些在黄海中南部越冬的鱼类洄游至 此产卵繁殖。同时本研究也发现, 5月、6月航次调 查的渔获中出现了大量许氏平鲉和大泷六线鱼幼 鱼，这些都可能导致春季鱼类生物量明显多于其他 季节; 秋季水温降低, 许氏平鲉等一些冷温性种生 长迅速, 使得秋季鱼类生物量也较高。而7、8月份 崂山湾海域正处于 $22.68-25.84^{\circ} \mathrm{C}$ 的高温期, 大型藻
类衰败分解需消耗大量的溶解氧，礁区和对照区处 于低氧状态 (8月调查海域平均溶解氧仅为 4.22 $\mathrm{mg} / \mathrm{L}$ ), 在一定程度上会影响水生生物的生长(祁铭 华等, 2004)。同时，该季节汻苔和石莼类大量暴发， 有些会堵塞调查用地笼网网口, 使得渔获效率降 低。冬季海域温度降低, 大部分鱼类迁徙至深水区, 因而冬季的渔获量也较少。

\section{3 游泳动物群落扰动}

丰度/生物量比较法由Warwick (1986)最先提 
出, 它最初主要通过比较生物量和丰度的优势地位 来监测污染对底栖动物群落的影响。随后, 被应用 到不同区域海洋底栖动物群落研究中, 而且在大多 数情况下可以显示出扰动产生的预期影响(Warwick \& Pearson, 1987; Agard et al, 1993), 近些年亦被应 用于游泳动物群落研究(Yemane et al, 2005)。当生物 量曲线位于丰度曲线上方 ( $W$ 统计为正值) 时, 说明 群落中生物量分布比丰度分布更具优势, 群落的生 物量是由一种或几种大型的种类控制; 当丰度曲线 位于生物量曲线上方 ( $W$ 统计为负值) 时, 说明群落 中丰度分布比生物量分布更具优势, 群落的丰度是 由一种或者几种小个体种类控制。本研究发现, 春 季和夏初崂山湾底层游泳动物群落处于严重受扰 动状态, 秋末和冬季底层游泳动物群落处于稳定状 态, 其他月份则处于中度扰动状态, 推测是由于优 势种以及优势种的个体大小改变引起的。春季和夏 初, 鱼类优势种主要以大泷六线鱼、许氏平鲉的幼 鱼为主, 其生物量占到总渔获量的 $40 \%$ 以上, 数量 则占到 $50 \%$ 以上，个体的平均体重分别达到 $22.26 \mathrm{~g}$ 和 $46.34 \mathrm{~g}$; 同时, 5、6月份调查海域还出现了大量的 寄居蟹, 个体数占到总数的 $10 \%$ 以上, 但生物量仅 占总渔获量的 $2 \%$ 。因此, 该季节丰度比生物量更具 优势地位, 丰度曲线位于生物量曲线上方; 而到了 11 月和次年的 1 月, 大泷六线鱼和许氏平鮋的生物 量占到总渔获量的 $70 \%$ 以上, 数量占到 $50 \%$ 以上, 个体的平均体重也分别达到 $54.15 \mathrm{~g}$ 和 $112.08 \mathrm{~g}$, 生 物量比丰度更具优势地位, 生物量曲线位于丰度曲 线的上方。各月份优势种类以及优势种个体大小的 差异, 使生物量曲线和丰度曲线的支配地位不同, 因而由 $\mathrm{ABC}$ 曲线所呈现出的群落稳定性存在一定 差异。

\section{4 游泳动物群落多样性}

各月份人工鱼礁区和对照区底层游泳动物群 落的种类丰富度指数、多样性指数和均匀度指数均 无显著差异。分析认为, 鱼礁区和对照区都位于近 岸水域, 大型藻类丰富, 水深较浅, 饵料生物丰富, 栖息地的复杂程度较高, 游泳动物种类相似, 从而 导致鱼礁区和对照区游泳群落的多样性、丰富度和 均匀度无明显差异。 $8 、 9$ 月份底层游泳动物群落的 种类丰富度指数、多样性指数和均匀度指数均较高, 游泳动物群落结构较为复杂。11月和 1 月的丰富度 指数和多样性指数偏低, 推测可能与游泳动物种类
数减少和优势种组成变化有关。秋末水温降低,一 些种类前往深水区越冬, 导致海域游泳动物种类数 减少。此外，这两个月优势种许氏平鲉的质量百分 比均高达 $60 \%$ 以上，数量百分比也达到了 $40 \%$, 而 其余种类的数量和质量占比均比较少, 种类分布不 均匀, 导致游泳动物群落多样性和丰富度下降。

\section{5 游泳动物群落相似性分析}

底层游泳动物群落的SIMPER相似性百分比分 析表明, 各区域不同月份的游泳动物的组内相似性 较高，均达到 $55 \%$ 以上，而组间相异性则在 $50 \%$ 以 下, 表明各调查区域各月份游泳动物群落的相似性 较高, 对组内相似性和组间相异性贡献率最高的两 种游泳动物为日本䌧和许氏平鲉。分析认为调查区 域出现的游泳动物均为我国黄渤海常见种类, 日本 蟳、星康吉鳗、鲉形目和鲽形目等生物量占比较大 的一些种类, 均属于全年性岩礁种类 (张迎秋, 2015), 各月份均有出现, 使得调查区周年底层游泳 动物群落的相似性较高。聚类分析结果表明，5、6 月和 11 月调查区的游泳动物样方聚为一支, 即春季 和秋季底层游泳动物群落的相似性最高, 与荣成俚 岛人工鱼礁区聚类结果一致(吴忠金等, 2012)。分析 认为, 春季随着水温不断回升, 一些种类开始向近 岸产卵场洄游; 秋季随水温逐渐下降, 沿岸鱼类的 越冬和索饵行为增强, 活动范围增大, 从而导致 春、秋季底层游泳动物群落结构的趋同。

本研究表明, 崂山湾人工鱼礁投放后, 鱼礁区 游泳动物的种类数和生物量均高于对照区, 鱼礁的 投放在一定程度上增加了海域资源量, 改变了游泳 动物群落结构。但人工鱼礁生态效果的显现是一个 长期、渐进的过程, 现有数据只能对人工鱼礁的生 态效果做出初步评价, 今后还要对调查海域进行长 期持续的监测研究, 采用多种网具相结合的方式, 以期更全面准确地反映当地渔业资源的组成。此外, 应重点关注礁区生态系统的稳定性、渔获质量、主 要渔获种类的食物关系以及礁区水动力机制等, 利 用环境容纳量、生态系统服务价值等方法, 深入评 估人工鱼礁的生态效应, 为人工鱼礁生态系统的可 持续开发利用提供科学依据。

\section{参考文献}

Agard JBR, Gobin J, Warwick RM (1993) Analysis of marine macrobenthic community structure in relation to pollution, natural oil seepage and seasonal disturbance in a tropical 
environment (Trinidad, West Indies). Marine Ecology Progress Series, 92, 233-243.

Bray JR, Curtis JT (1957) An ordination of the upland forest communities of southern Wisconsin. Ecological Monographs, 27, 325-349.

Chen Y, Yu CQ, Zhang GS, Zhang S (2002) The environment function and fish gather effect of artificial reefs. Journal of Dalian Fisheries University, 17, 64-69. (in Chinese with English abstract) [陈勇, 于长清, 张国胜, 张硕 (2002) 人 工鱼礁的环境功能与集鱼效果. 大连水产学院学报, 17 , 64-69.]

Cheng QT, Zheng BS (1987) Systematic Synopsis of Chinese Fishes. Science Press, Beijing. (in Chinese) [成庆泰, 郑葆 珊 (1987) 中国鱼类系统检索. 科学出版社, 北京.]

Clarke KR, Warwick RM (2001) Change in Marine Communities: An Approach to Statistical Analysis and Interpretation, 2nd edn. PRIMER-E, Plymouth.

Ji DP, Bian XD, Song N, Gao TX (2014) Feeding ecology of Hexagrammos otakii in Lidao Rongcheng. Journal of Fisheries of China, 38, 1399-1409. (in Chinese with English abstract) [纪东平, 市晓东, 宋娜, 高天翔 (2014) 荣成俚岛 大泷六线鱼摄食生态研究. 水产学报, 38, 1399-1409.]

Ji DP, Bian XD, Song N, Gao TX (2015) Feeding ecology of Hexagrammos agrammus in Lidao, Rongcheng, China. Journal of Fishery Sciences of China, 22, 88-98. (in Chinese with English abstract) [纪东平, 市晓东, 宋娜, 高天翔 (2015) 荣成俚岛斑头鱼摄食生态研究. 中国水产科学, 22, 88-98.]

Jiao JJ, Pan YX, Sun LY, Yang BQ, Qiu SY (2011) Effect of artificial reefs on fish multiplication. Fisheries Science, 30(2), 79-82. (in Chinese with English abstract) [焦金菊, 潘永告, 孙利元, 杨宝清, 邱盛尧 (2011) 人工鱼礁区的 增殖鱼类资源效果初步探究. 水产科学, 30(2), 79-82.]

Li F, Zhang HJ, Lü ZB, Xu BQ, Zheng L (2013) Species composition and community diversity of nekton in Laizhou Bay, China. Biodiversity Science, 21, 537-546. (in Chinese with English abstract) [李凡, 张焕君, 吕振波, 徐炳庆, 郑亮 (2013) 莱州湾游泳动物群落种类组成及多样性. 生物多 样性, 21, 537-546.]

Lin J, Zhang SY (2006) Research advances on physicial stability and ecological effects of artificial reef. Marine Fisheries, 28, 257-262. (in Chinese with English abstract) [林军, 章 守宇 (2006) 人工鱼礁物理稳定性及其生态效应的研究 进展. 海洋渔业, 28, 257-262.]

Liu J, Ning P (2011) Species composition and faunal characteristics of fishes in the Yellow Sea. Biodiversity Science, 19, 764-769. (in Chinese with English abstract) [刘静, 宁 平 (2011) 黄海鱼类组成、区系特征及历史变迁. 生物多 样性, 19, 764-769.]

Liu RY (2008) Checklist of Marine Biota of China Seas. Science Press, Beijing. (in Chinese) [刘瑞玉 (2008) 中国海洋 生物名录. 科学出版社, 北京.]

Ludwig JA, Reynolds JF (1988) Statistical Ecology: A Primer on Methods and Computing. John Wiley and Sons, New York.

Ma ZY, Wang HQ (1997) Multivariate analysis of community structure on macrobenthos. China Environmental Science, 17, 297-300. (in Chinese with English abstract) [马藏允, 王惠卿 (1997) 底栖生物群落结构变化多元变量统计分 析. 中国环境科学, 17, 297-300.]

Margalef R (1968) Perspectives in Ecological Theory. University of Chicago Press, Chicago.

Mei C, Ren YP, Xu BD, Fan YC (2010) Preliminary study on the effect of Penaeus japonicas Bate releasing in the Laoshan Bay. Periodical of Ocean University of China (Natural Science Edition), 40(9), 45-50. (in Chinese with English abstract) [梅春, 任一平, 徐宾铎, 范延琛 (2010) 崂山湾日本对虾增殖放流效果的初步研究. 中国海洋大 学学报(自然科学版), 40(9), 45-50.]

Palomares MLD, Pauly D (2015) SeaLifeBase. http://www. sealifebase.org. (accessed on 2015-03-25)

Pielou EC (1966) Species-diversity and pattern-diversity in the study of ecological succession. Journal of Theoretical Biology, 10, 370-383.

Pinkas L, Olphant M, Iverson I (1971) Food habits of albacore, bluefin tuna and bonito in California waters. California Department of Fish and Game: Fish Bulletin, 152, 1-105.

Qi MH, Ma SS, Qu KM, Xin FY (2004) The formation of sulfide in the marine sediments and its relationships to cultivation of shellfish. Marine Fisheries Research, 25(1), 85-89. (in Chinese with English abstract) [祁铭华, 马绍赛, 曲克 明, 辛福言 (2004) 沉积环境中硫化物的形成及其与贝 类养殖的关系. 海洋水产研究, 25(1), 85-89.]

Rounsefell GA (1972) Ecological effects of offshore construction. Journal of Marine Science, 2, 1-119.

Tang YL, Sheng HX, Qi GR, Wu LH, Wan R (2012) The seasonal differences of benthic macroalgae communities in three artificial reefs of Laoshan Bay. Fisheries Modernization, 39(3), 66-71. (in Chinese with English abstract) [唐衍 力, 盛化香, 齐广瑞, 吴连慧, 万荣 (2012) 崂山湾三个 人工鱼礁区底栖大型藻类群落的季节性差异. 渔业现代 化, 39(3), 66-71.]

Wang X, Sheng HX, Tang YL, Huang LY, Wan R (2014) Relationship between the structure of phytoplankton community and environmental factors in three artificial reef areas of Laoshan Bay. Progress in Fishery Sciences, 35(4), 7-12. (in Chinese with English abstract) [王欣, 盛化香, 唐衍力, 黄 六一, 万荣 (2014) 崂山湾人工鱼礁区浮游植物群落结 构与环境因子的关系. 渔业科学进展, 35(4), 7-12.]

Wang ZH, Zhang SY, Chen QM, Xu Q, Wang K (2012) Fish community ecology in rocky reef habitat of Ma'an Archipelago. I. Species composition and diversity. Biodiversity Science, 20, 41-50. (in Chinese with English abstract) [汪振 华, 章守宇, 陈清满, 许强, 王凯 (2012) 马鞍列岛岩礁 生境鱼类群落生态学. I. 种类组成和多样性. 生物多样 性, 20, 41-50.] 
Warwick RM (1986) A new method for detecting pollution effects on marine macrobenthic communities. Marine Biology, 92, 557-562.

Warwick RM, Pearson TH (1987) Detection of pollution effects on marine macrobenthos: further evaluation of the species abundance/biomass method. Marine Biology, 95, 193-200.

Wu ZX, Zhang L, Zhang XM, Zhang PD, Li WT (2012) Nekton community structure and its relationship with main environmental variables in Lidao artificial reef zones of Rongcheng. Acta Ecologica Sinica, 32, 6737-6746. (in Chinese with English abstract) [ 吴忠金錪, 张否, 张秀梅, 张 沛东, 李文涛 (2012) 荣成俚岛人工鱼礁区游泳动物群 落特征及其与主要环境因子的关系. 生态学报, 32, 6737-6746.]

Xu BQ, Lü ZB, Li F, Bao JG, Xu WZ (2011) On nekton composition in southern coastal waters off Shandong Peninsula in summer. Marine Fisheries, 33, 59-65. (in Chinese with English abstract) [徐炳庆, 吕振波, 李凡, 包家国, 徐维柱 (2011) 山东半岛南部近岸海域夏季游泳动物的组成特 征. 海洋渔业, 33, 59-65.]

Xu H, Zeng XQ, Gu YB, Guan LS (2012) The effect of artificial reefs on the community structure and seasonal variation of nektons in Zhuwang Harbor, Laizhou, Shandong. Periodical of Ocean University of China (Natural Science Edition), 42(5), 47-54. (in Chinese with English abstract) [徐 浩, 曾晓起, 顾炎斌, 关丽莎 (2012) 人工鱼礁对山东莱 州朱旺港海区游泳动物的群落结构及季节变化的影响. 中国海洋大学学报(自然科学版), 42(5), 47-54.]

Yang DJ, Wang YL (1989) Invertebrates in North China. Higher Education Press, Beijing. (in Chinese) [杨德渐, 王 永良 (1989) 中国北部无脊椎动物. 高等教育出版社, 北 京.]

Yemane D, Field JG, Leslie RW (2005) Exploring the effects of fishing on fish assemblages using Abundance Biomass Comparison $(\mathrm{ABC})$ curves. ICES Journal of Marine Science, 62, 374-379.

Yu Q, Tang YL (2015) Analysis on community structure and diversity of fish and macroinvertebrates in artificial reef area around Xigang, Weihai. Fishery Modernization, 42(3), 65-72. (in Chinese with English abstract) [于晴, 唐衍力 (2015) 威海西港人工鱼礁区鱼类和大型无脊椎动物群聚 特征. 渔业现代化, 42(3), 65-72.]

Yuan W, Lin Q, Wang J, Sun JQ, Chen RS (2015) Assessment of enhancement effectiveness of Chinese shrimp Fenneropenaeus chinensis stock in the Laoshan Bay. Progress in Fishery Sciences, 36(4), 27-34. (in Chinese with English abstract) [袁伟，林群，王俊，孙坚强，陈瑞盛（2015）崂 山湾中国对虾(Fenneropenaeus chinensis)增殖放流效果评 价. 渔业科学进展, 36(4), 27-34.]

Zhang B, Yuan W, Wang J (2015) Feeding ecology of the dominant fish species in spring in Laoshan Bay. Journal of Fishery Sciences of China, 22, 820-827. (in Chinese with English abstract) [张波, 袁伟, 王俊 (2015) 崂山湾春季 鱼类群落的摄食生态及其主要种类. 中国水产科学, 22 , 820-827.]

Zhang S, Sun MC, Chen Y (2008) The attractive effects of different structural artificial reef models on juvenile Schlegel's rockfish Sebastes schlegeli and fat greenling Hexagrammos otakii. Journal of Dalian Fisheries University, 23, 13-19. (in Chinese with English abstract) [张硕, 孙满昌, 陈勇 (2008) 人工鱼礁模型对大泷六线鱼和许氏平鲉幼 鱼个体的诱集效果. 大连水产学院学报, 23, 13-19.]

Zhang YQ (2015) Community Structure, Feeding Ecology and Movement Behaviors of Demersal Fish around Qiansan Islets. $\mathrm{PhD}$ dissertation, University of Chinese Academy of Sciences, Beijing. (in Chinese with English abstract) [张迎 秋 (2015) 前三岛海域底层鱼类群落结构、摄食生态和运 动行为特征. 博士学位论文, 中国科学院大学, 北京.]

Zhou YB, Chen PM, Li HQ (2011) Feasibility study on artificial reef construction in planning area around Liuniu of Zhelin Bay in Guangdong Province. Guangdong Agricultural Sciences, 38(23), 10-13. (in Chinese with English abstract) [周艳波，陈丕茂，李辉权 (2011) 广东省柘林湾海 域溜牛礁区建礁可行性研究.广东农业科学, 38(23), 10-13.]

(责任编委: 李新正＼cjkstart责任编辑：闵文杰)

\section{附录 Supplementary Material}

附录12015年5月至2016年1月崂山湾人工鱼礁区游泳动物名录

Appendix 1 The list of caught nektons in the artificial reef zones in Laoshan Bay from May 2015 to January 2016 http:// www.biodiversity-science.net/fileup/PDF/2016111-1.pdf 
刘鸿雁, 杨超杰, 张沛东, 李文涛, 杨晓龙, 张秀梅. 青岛崂山湾人工鱼礁区底层游泳动物群落结构特征. 生物多样性, 2016, 24 (8): 896-906. http://www.biodiversity-science.net/CN/10.17520/biods.2016111

附录1２015年5月至2016年1月崂山湾人工鱼礁区游泳动物名录

Appendix 1 The list of caught nektons in the artificial reef zones in Laoshan Bay from May 2015 to January 2016

\begin{tabular}{|c|c|c|c|c|c|c|c|c|c|c|}
\hline \multirow{2}{*}{ 种类 Species } & \multicolumn{2}{|c|}{ 生态类型 Eco-type } & \multicolumn{8}{|c|}{ 调查时间 Survey time } \\
\hline & ST & HT & 2015.05 & 2015.06 & 2015.07 & 2015.08 & 2015.09 & 2015.10 & 2015.11 & 2016.01 \\
\hline \multicolumn{11}{|l|}{ 鲉科 Scorpaenidae } \\
\hline 许氏平鲉 Sebastes schlegelii & CT & $\mathrm{CD}$ & * & * & * & * & * & * & * & * \\
\hline 厚头平鲉 S. pachycephalus & WT & $\mathrm{CD}$ & * & * & & * & * & * & * & * \\
\hline 褐菖鲉 Sebastiscus mamoratus & WT & $\mathrm{CD}$ & * & * & * & * & * & * & * & * \\
\hline 朝鲜平鲉 Sebastes koreanus & WT & $\mathrm{CD}$ & * & * & * & * & * & * & * & * \\
\hline \multicolumn{11}{|l|}{ 六线鱼科 Hexagrammidae } \\
\hline 斑头鱼 Hexagrammus agrammus & СT & $\mathrm{CD}$ & * & * & * & $*$ & * & * & * & * \\
\hline 大泷六线鱼 H. otakii & СТ & $\mathrm{CD}$ & * & * & * & * & * & * & * & * \\
\hline \multicolumn{11}{|l|}{ 虾虎鱼科 Gobiidae } \\
\hline 矛尾虾虎鱼 Chaeturichthys stigmatias & WT & $\mathrm{CD}$ & & & & & & & * & * \\
\hline 五带高鯺虾虎鱼 Pterogobius zacalles & WT & $\mathrm{CD}$ & & * & & $*$ & * & & & \\
\hline 纹稿虾虎鱼 Tridentiger trigonocephalus & WT & $\mathrm{CD}$ & & & & * & * & & * & * \\
\hline 六丝针尾虾虎鱼 Amblychaeturichthys hexanema & WT & $\mathrm{CD}$ & & * & & & & & & \\
\hline 斑尾复虾虎鱼 Synechogobius ommaturus & WT & $\mathrm{CD}$ & & & & & * & * & & \\
\hline 钟道虾虎鱼 Tridentiger barbatus & WT & $\mathrm{CD}$ & & & & & & * & & * \\
\hline \multicolumn{11}{|l|}{ 石首鱼科 Sciaenidae } \\
\hline 黄姑鱼 Nibea albiflora & WT & $\mathrm{CBD}$ & & & & * & $*$ & * & & \\
\hline 皮氏叫姑鱼 Johnius belengeri & WT & CBD & * & & * & * & & & * & \\
\hline 小黄鱼 Pseudosciaena Polyactis & WT & CBD & & & & & * & & & \\
\hline 银姑鱼 Pennahia argentata & WT & CBD & & & & & * & & & \\
\hline \multicolumn{11}{|l|}{ 锦䲁科 Pholidae } \\
\hline 方氏云䲁 Pholis fangi & $\mathrm{CT}$ & $\mathrm{CD}$ & * & * & & & * & * & * & \\
\hline 云䲁 P. nebulosus & $\mathrm{CT}$ & $\mathrm{CD}$ & & & & * & & & * & * \\
\hline 繸䲁 Chirolophis japonicus & $\mathrm{CT}$ & $\mathrm{CD}$ & * & * & & & & & & * \\
\hline \multicolumn{11}{|l|}{ 绵㬠科 Zoarcidae } \\
\hline 绵解 Zoarces elontatus & $\mathrm{CT}$ & $\mathrm{CD}$ & * & & & & & & & \\
\hline \multicolumn{11}{|l|}{ 鱚科 Sillaginidae } \\
\hline 少鳞鱚 Sillago japonica & WT & CD & & & & * & & & & \\
\hline \multicolumn{11}{|l|}{ 鲷科 Sparidae } \\
\hline 黑鲷 Sparus macrocephlus & WT & $\mathrm{CD}$ & * & & & & & * & & \\
\hline \multicolumn{11}{|l|}{ 天竺鯛科 Apogonidae } \\
\hline 细条天竺鱼 Apogonichthys lineatus & WT & $\mathrm{CD}$ & & & * & & & & & \\
\hline \multicolumn{8}{|l|}{ 真鲇科 Percichthyidae } & & & \\
\hline 花鲇 Lateolabrax maculatus & WT & CRA & & & * & & * & * & * & \\
\hline \multicolumn{11}{|l|}{ 鲬科 Platycephalidae } \\
\hline 褐斑鲬 Platycephalus sp.1 & WT & CRA & & & & $*$ & & & * & \\
\hline \multicolumn{11}{|l|}{ 鲂鮄科 Triglidae } \\
\hline 小眼绿鯺鱼 Chelidonichthys spinosus & WT & CD & & & & & & * & & \\
\hline \multicolumn{11}{|l|}{ 康吉鳗科 Congridae } \\
\hline 星康吉鳗 Conger myriaster & WT & CD & * & * & * & * & * & * & * & * \\
\hline \multicolumn{11}{|l|}{ 牙鲆科 Paralichthyidae } \\
\hline 褐牙鲆 Paralichthys olivaceus & WT & $\mathrm{CD}$ & & & * & * & * & & & \\
\hline \multicolumn{11}{|l|}{ 鲽科 Pleuronectidae } \\
\hline 钝吻黄盖鲽 Pleuronectes Vokohamae & СТ & $\mathrm{CD}$ & * & & * & & & & & \\
\hline 石鲽 Kareius bicoloratus & СТ & $\mathrm{CD}$ & $*$ & * & * & * & & * & & * \\
\hline 舌鲳科 Cynoglossidae & & & & & & & & & & \\
\hline 褐斑三线舌鲳 Cynoglossus trigrammus & WT & $\mathrm{CD}$ & & * & & & & & & \\
\hline 长吻红舌鲳 C. lighti & WT & $\mathrm{CD}$ & * & & * & * & $*$ & & & \\
\hline 半滑舌鳎 C. semilaevis & WT & $\mathrm{CD}$ & $*$ & & & & & & & \\
\hline 短吻红舌鳎 C. joyneri & WT & $\mathrm{CD}$ & & & * & & * & & & \\
\hline
\end{tabular}




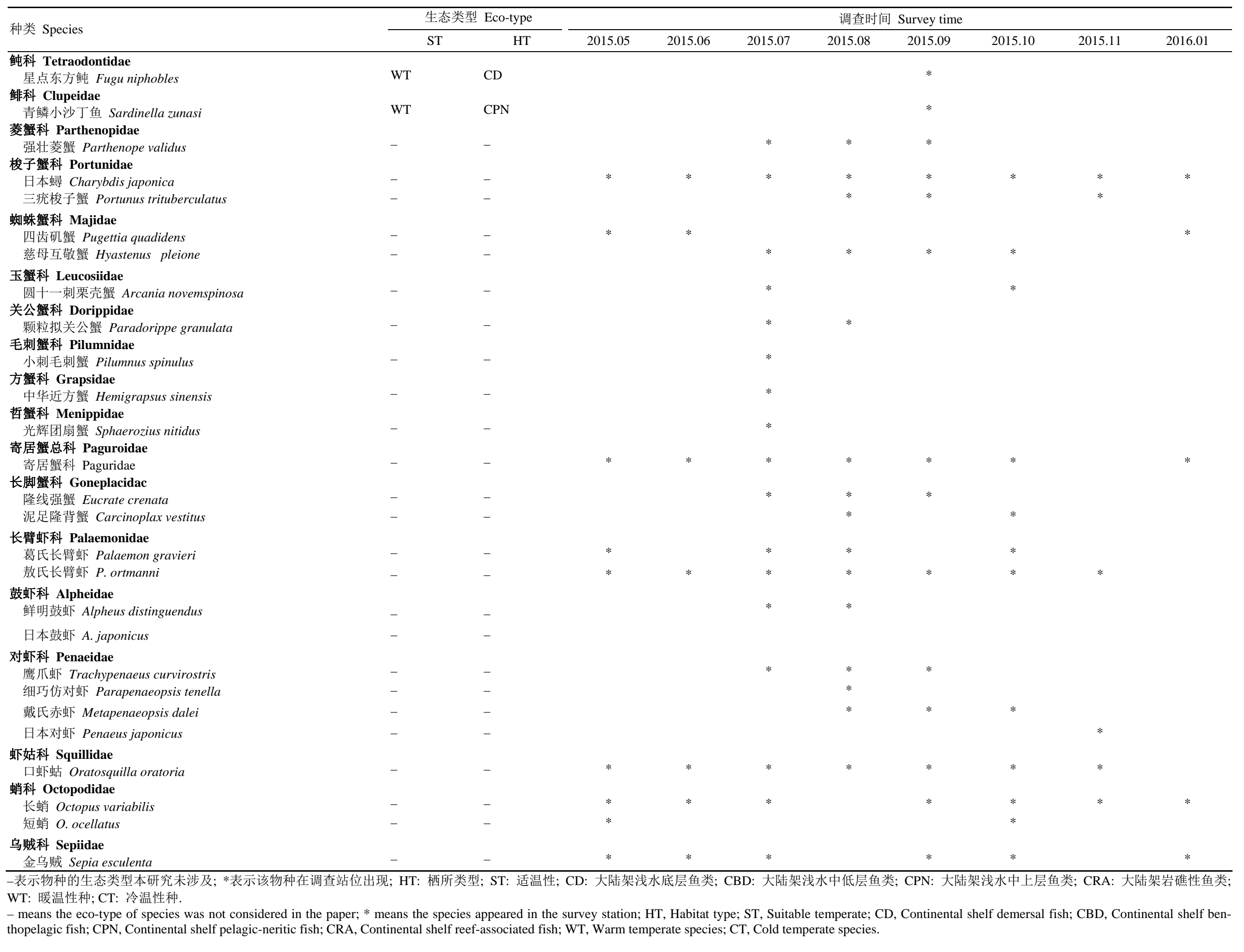

